\title{
Exploring Non-Orthosteric Interactions with a Series of Potent and Selective A Antagonists
}

Darío Miranda-Pastoriza, ${ }^{1,2}$ Rodrigo Bernardez, ${ }^{1,2}$ Jhonny Azuaje, ${ }^{1,2}$ Rubén Prieto-Díaz, ${ }^{1,2,3}$ Maria Majellaro, ${ }^{1,2}$ Ashish V. Tamhankar, ${ }^{3}$ Lucien Koenekoop, ${ }^{3}$ Alejandro González, ${ }^{4}$ Claudia Gioé-Gallo, ${ }^{1,2}$ Ana Mallo-Abreu, ${ }^{1,2}$ José Brea, ${ }^{4 *}$ M. Isabel Loza, ${ }^{4}$ Aitor García-Rey, ${ }^{1,2}$ Xerardo García-Mera, ${ }^{2}$ Hugo Gutiérrez-de-Terán, ${ }^{3 *}$ and Eddy Sotelo ${ }^{1,2^{*}}$

${ }^{1}$ Centro Singular de Investigación en Química Biolóxica e Materiais Moleculares (CIQUS) and ${ }^{2}$ Departamento de Química Orgánica, Universidade de Santiago de Compostela, 15782 Santiago de Compostela, Spain. ${ }^{3}$ Department of Cell and Molecular Biology, SciLifeLab, Uppsala University, Uppsala SE-75124. ${ }^{4}$ Centro Singular de Investigación en Medicina Molecular y Enfermedades Crónicas (CIMUS). Universidade de Santiago de Compostela, 15782. Santiago de Compostela, Spain.

\section{TABLE OF CONTENTS}

Chemistry. General information. S2

General pathways for synthesis and spectroscopic and analytical data for compounds described. . $\mathrm{S} 3$

Pharmacology. Binding assays S1 1

HPLC traces of best compounds. S12

References. S15 


\section{Chemistry. General information}

Commercially available starting materials, reagents and solvents were purchased and used without further purification. The reactions were monitored by thin-layer chromatography (TLC) with $2.5 \mathrm{~mm}$ Merck silica gel GF 254 strips, and the purified compounds each showed a single spot; unless stated otherwise, UV light, $p$-anisaldehyde solution and/or iodine vapor were used for detection of compounds. The Ugi reactions were performed in coated Kimble vials on a PLS (6X4) Organic Synthesizer with orbital stirring. Purity and identity of all tested compounds were established by a combination of mass spectrometry, HRMS and NMR spectra as described below. Purification of isolated products was carried out by column chromatography (Kieselgel 0.040-0.063 mm, E. Merck) or medium pressure liquid chromatography (MPLC) on a ComBiFlash Companion (Teledyne ISCO) with RediSep pre-packed normal-phase silica gel $(35-60 \mu \mathrm{m})$ columns. Melting points were determined on a Stuart Scientific melting point apparatus and are uncorrected.

The NMR spectra were recorded on Bruker AM300 and XM500 spectrometers. Chemical shifts are given as $\delta$ values against tetramethylsilane as internal standard and $\mathbf{J}$ values are given in $\mathrm{Hz}$. Mass spectra were obtained on a Varian MAT-711 instrument. High-resolution mass spectra were obtained on an Autospec Micromass spectrometer. The purity of all tested compounds was determined to be $>95 \%$. A detailed description of synthetic methodologies as well as analytical and spectroscopic data for all described compounds is described below. 


\section{Synthesis, spectroscopic and analytical data of compounds described:}

General procedure for the synthesis of 2-(2-((2,6-bis(4-methoxyphenyl)pyrimidin-4-yl)amino)2-oxoethoxy)acetic acid (3a) and 4-((2,6-bis(4-methoxyphenyl)pyrimidin-4-yl)amino)-4oxobutanoic acid (3b).

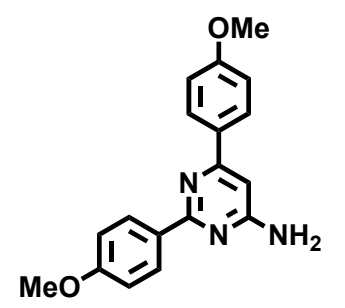

1
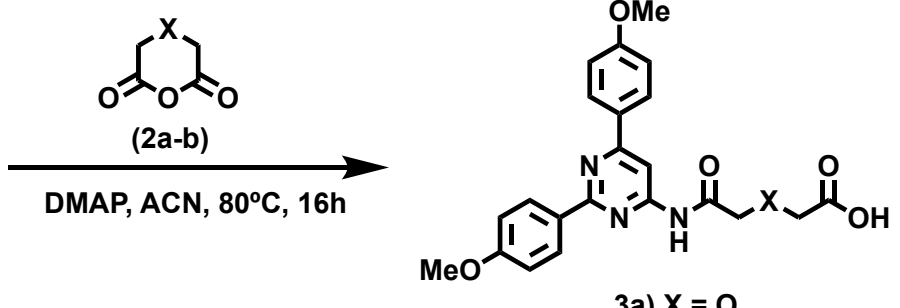

3a) $X=0$

$3 b X=-$

A mixture of 2,6-bis(4-methoxyphenyl)pyrimidin-4-amine ${ }^{1} 1$ (1 equiv.), diglycolic $2 \mathbf{a}$ or succinic anhydride $\mathbf{2 b}$ (1.5 equiv.) and 4-dimethylaminopyrimidine (DMAP, 0.1 equiv.) in acetonitrile (ACN, $5 \mathrm{~mL}$ ) was stirred at $80^{\circ} \mathrm{C}$ for $16 \mathrm{~h}$. After completion of the reaction, the solvent was evaporated under vacuum to afford an oily residue that was purified by chromatographic methods on silica gel using $\mathrm{CH}_{2} \mathrm{Cl}_{2} / \mathrm{MeOH}$ mixture.

2-(2-((2,6-bis(4-methoxyphenyl)pyrimidin-4-yl)amino)-2-oxoethoxy)acetic acid (3a). Yield: 61\%. Mp. $195-197^{\circ} \mathrm{C} .{ }^{1} \mathrm{H}$ NMR (300 MHz, DMSO- $\left.d_{6}\right) \delta(\mathrm{ppm}): 12.86(\mathrm{~s}, 1 \mathrm{H}), 10.56(\mathrm{~s}, 1 \mathrm{H}), 8.46-8.36$ $(\mathrm{m}, 2 \mathrm{H}), 8.32(\mathrm{~s}, 1 \mathrm{H}), 8.20-8.11(\mathrm{~m}, 2 \mathrm{H}), 7.17-7.04(\mathrm{~m}, 4 \mathrm{H}), 4.33(\mathrm{~s}, 2 \mathrm{H}), 4.23(\mathrm{~s}, 2 \mathrm{H}), 3.84(\mathrm{~s}$, 6H). MS (CI) $m / z(\%): 424.1\left([\mathrm{M}+\mathrm{H}]^{+}, 100\right)$.

4-((2,6-bis(4-methoxyphenyl)pyrimidin-4-yl)amino)-4-oxobutanoic acid (3b). Yield: 58\%. Mp. 205 $207{ }^{\circ} \mathrm{C} .{ }^{1} \mathrm{H}$ NMR $\left(300 \mathrm{MHz}, \mathrm{DMSO}-d_{6}\right) \delta(\mathrm{ppm}): 12.19$ (brs, $\left.1 \mathrm{H}\right), 10.93$ (brs, $\left.1 \mathrm{H}\right), 8.49-8.39(\mathrm{~m}$, 2H), $8.36(\mathrm{~s}, 1 \mathrm{H}), 8.21-8.12(\mathrm{~m}, 2 \mathrm{H}), 7.17-7.06(\mathrm{~m}, 4 \mathrm{H}), 3.86(\mathrm{~s}, 6 \mathrm{H}), 2.75(\mathrm{t}, J=6.6 \mathrm{~Hz}, 2 \mathrm{H})$, $2.60-2.53(\mathrm{~m}, 2 \mathrm{H}) . \mathrm{MS}(\mathrm{CI}) \mathrm{m} / z(\%): 408.1\left([\mathrm{M}+\mathrm{H}]^{+}, 100\right)$. 
General procedure for the synthesis of $\mathrm{N}_{1}-(2,6-b i s(4-m e t h o x y p h e n y l) p y r i m i d i n-4-y l)-\mathrm{N}_{4}-(2-$ (alkylamino)-2-oxoethyl)succinamides (7a-f) and N-(2,6-bis(4-methoxyphenyl)pyrimidin-4-yl)2-(2-((2-(alkylamino)-2-oxoethyl)amino)-2-oxoethoxy)acetamides (8a-f)

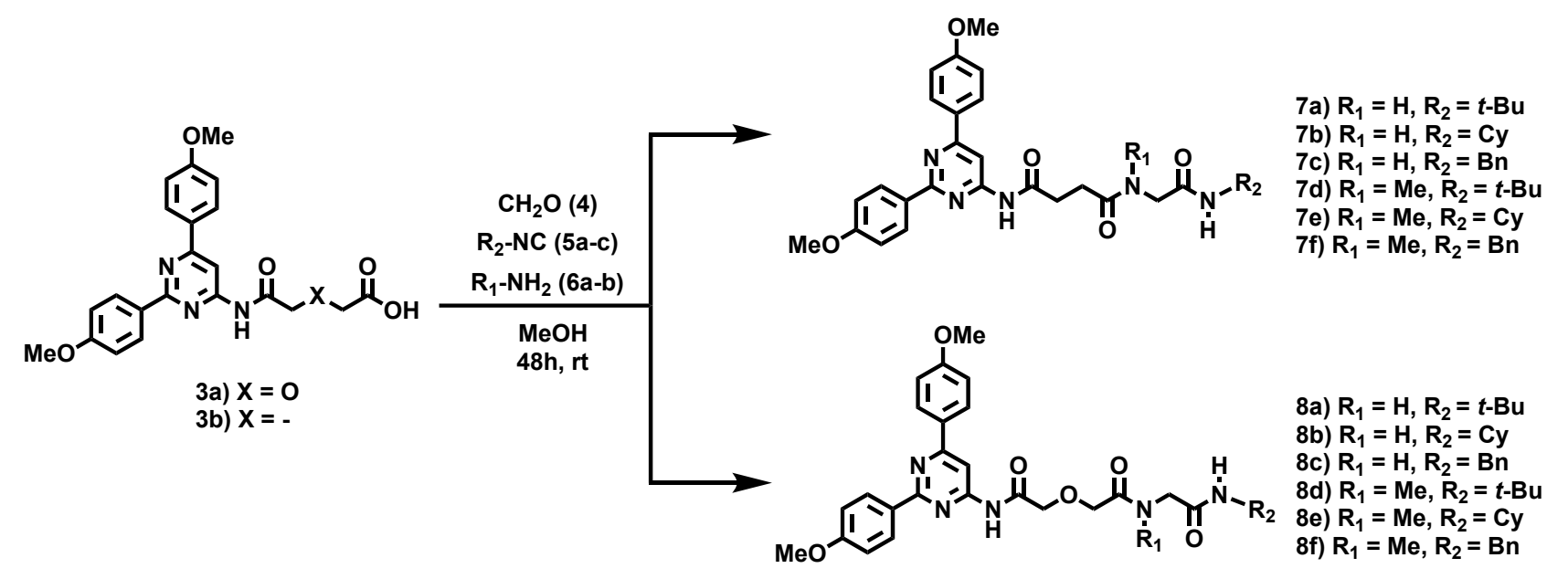

A mixture of the synthetised acid 3a-b (1 equiv.), formaldehyde 4 ( 1 equiv.), the isocyanide 5a-c (1 equiv.) and the amine 6a-b (1 equiv) in $\mathrm{MeOH}(3 \mathrm{~mL})$ was submitted to orbital stirring at room temperature for $48 \mathrm{~h}$. After completion of the reaction, $\mathrm{CH}_{2} \mathrm{Cl}_{2}(3 \mathrm{~mL})$ and PS- $p$-TsOH (2 equiv.) were added. The reaction mixture was submitted to orbital stirring at room temperature until complete consumption of unreacted isocyanide $(30-60 \mathrm{~min})$. The polymeric reagent was filtered off and successively washed [2 times $(4 \mathrm{~mL})]$ with $\mathrm{MeOH}$, AcOEt and $\mathrm{CH}_{2} \mathrm{Cl}_{2}$. Evaporation of the solvents from the filtrate afforded a residue, treated with water and extracted with ethyl acetate [3 times (15 $\mathrm{mL})]$. The organic phase was dried $\left(\mathrm{Na}_{2} \mathrm{SO}_{4}\right)$ and evaporated under reduced pressure to afford an oily residue that was purified by chromatographic methods on silica gel using hexane/AcOEt mixtures. 
$N_{1^{-}}$(2,6-bis(4-methoxyphenyl)pyrimidin-4-yl)- $N_{4^{-}}$(2-(tert-butylamino)-2-oxoethyl)succinamide (7a). Yield: $18 \%$. Mp. $176-178{ }^{\circ} \mathrm{C} .{ }^{1} \mathrm{H}$ NMR $\left(300 \mathrm{MHz}, \mathrm{CDCl}_{3}\right) \delta(\mathrm{ppm}): 8.44(\mathrm{~d}, J=8.5 \mathrm{~Hz}, 3 \mathrm{H}), 8.33$ (s, 1H), $8.18(\mathrm{dd}, J=9.1,3.5 \mathrm{~Hz}, 2 \mathrm{H}), 7.00(\mathrm{dd}, J=8.5,2.1 \mathrm{~Hz}, 4 \mathrm{H}), 3.88(\mathrm{~s}, 6 \mathrm{H}), 3.43-3.35(\mathrm{~m}$, 2H), $2.90(\mathrm{~s}, 2 \mathrm{H}), 2.73(\mathrm{~s}, 2 \mathrm{H}), 1.29(\mathrm{~s}, 9 \mathrm{H})$. MS (CI) $m / z(\%): 520.2\left([\mathrm{M}+\mathrm{H}]^{+}, 83\right)$.

$N_{l^{-}}\left(2,6-\right.$ bis(4-methoxyphenyl)pyrimidin-4-yl)-N $N_{4}-(2-(c y c l o h e x y l a m i n o)-2-o x o e t h y l)$ succinamide

(7b). Yield: 19\%. Mp. $202-204{ }^{\circ} \mathrm{C} .{ }^{1} \mathrm{H}$ NMR (300 MHz, $\left.\mathrm{CDCl}_{3}\right) \delta(\mathrm{ppm}): 8.65(\mathrm{~s}, 1 \mathrm{H}), 8.44(\mathrm{dd}, J$ $=8.9,1.9 \mathrm{~Hz}, 2 \mathrm{H}), 8.32(\mathrm{~d}, J=3.3 \mathrm{~Hz}, 1 \mathrm{H}), 8.22-8.09(\mathrm{~m}, 2 \mathrm{H}), 7.00(\mathrm{ddd}, J=9.0,4.2,2.0 \mathrm{~Hz}, 4 \mathrm{H})$, $3.88(\mathrm{~d}, J=2.1 \mathrm{~Hz}, 6 \mathrm{H}), 3.80-3.58(\mathrm{~m}, 1 \mathrm{H}), 3.38(\mathrm{~d}, J=3.1 \mathrm{~Hz}, 2 \mathrm{H}), 2.88(\mathrm{~d}, J=11.1 \mathrm{~Hz}, 2 \mathrm{H})$, $2.71(\mathrm{t}, J=5.7 \mathrm{~Hz}, 2 \mathrm{H}), 2.05-0.77(\mathrm{~m}, 10 \mathrm{H}) .{ }^{13} \mathrm{C} \mathrm{NMR}\left(75 \mathrm{MHz}, \mathrm{CDCl}_{3}\right) \delta(\mathrm{ppm}): 171.8,170.6$, $170.2,165.6,164.2,162.2,160.2,159.1,133.7,130.6,129.8,128.9,114.0,113.0,102.0,55.3,48.5$, 43.7, 32.5, 31.4, 31.0, 25.3, 24.6. MS (CI) $m / z(\%): 546.2\left([\mathrm{M}+\mathrm{H}]^{+}, 98\right)$.

$N_{l^{-}}$(2-(benzylamino)-2-oxoethyl)-N $N_{4}$ (2,6-bis(4-methoxyphenyl)pyrimidin-4-yl)succinamide (7c) Yield: $15 \%$. Mp. $184-186{ }^{\circ} \mathrm{C} .{ }^{1} \mathrm{H}$ NMR $\left(300 \mathrm{MHz}, \mathrm{CDCl}_{3}\right) \delta(\mathrm{ppm}): 8.65-8.31(\mathrm{~m}, 3 \mathrm{H}), 8.31-$ $8.00(\mathrm{~m}, 2 \mathrm{H}), 7.35-7.07(\mathrm{~m}, 5 \mathrm{H}), 7.07-6.84(\mathrm{~m}, 5 \mathrm{H}), 4.40(\mathrm{~d}, J=5.9 \mathrm{~Hz}, 2 \mathrm{H}), 3.87(\mathrm{~d}, J=2.4 \mathrm{~Hz}$, 6H), $3.34(\mathrm{~s}, 2 \mathrm{H}), 2.98-2.62(\mathrm{~m}, 4 \mathrm{H}) . \mathrm{MS}(\mathrm{CI}) \mathrm{m} / z(\%): 554.2\left([\mathrm{M}+\mathrm{H}]^{+}, 75\right)$.

$N_{1^{-}}\left(2,6-b i s(4-m e t h o x y p h e n y l)\right.$ pyrimidin-4-yl)- $N_{4-}$ (2-(tert-butylamino)-2-oxoethyl)- $N_{4^{-}}$ methylsuccinamide (7d). Yield: 22\%. Mp. $136-138{ }^{\circ} \mathrm{C} .{ }^{1} \mathrm{H}$ NMR (300 MHz, DMSO- $\left.d_{6}\right) \delta(\mathrm{ppm})$ : $10.87(\mathrm{~d}, J=1.9 \mathrm{~Hz}, 1 \mathrm{H}), 8.49-8.35(\mathrm{~m}, 2 \mathrm{H}), 8.34(\mathrm{~d}, J=2.1 \mathrm{~Hz}, 1 \mathrm{H}), 8.24-7.96(\mathrm{~m}, 2 \mathrm{H}), 7.62(\mathrm{~s}$, 1H), $7.17-7.00(\mathrm{~m}, 4 \mathrm{H}), 3.91(\mathrm{~s}, 2 \mathrm{H}), 3.84(\mathrm{~s}, 6 \mathrm{H}), 3.33-3.22(\mathrm{~m}, 3 \mathrm{H}), 2.99(\mathrm{~s}, 2 \mathrm{H}), 2.76(\mathrm{~s}, 2 \mathrm{H})$, $1.25(\mathrm{~d}, J=15.8 \mathrm{~Hz}, 9 \mathrm{H}) .{ }^{13} \mathrm{C}$ NMR $\left(75 \mathrm{MHz}, \mathrm{DMSO}-d_{6}\right) \delta(\mathrm{ppm}): 173.7,172.3,171.9,167.9,164.3$, $163.3,162.0,159.5,130.3,129.9,129.7,128.9,114.8,114.3,101.4,55.8,55.8,52.8,50.9,36.4,36.4$, 34.8, 34.8, 31.9, 29.0. HRMS (APCI) $m / z$ calcd for $\mathrm{C}_{29} \mathrm{H}_{36} \mathrm{~N}_{5} \mathrm{O}_{5}[\mathrm{M}+\mathrm{H}]^{+}:$534.2711; found: 534.2719. 
$N_{l^{-}}\left(2,6-\right.$ bis(4-methoxyphenyl)pyrimidin-4-yl)- $N_{4^{-}}(2-($ cyclohexylamino $)-2-o x o e t h y l)-N_{4}-m e t h y l-$ succinamide (7e). Yield: $21 \%$ Mp. $223-225^{\circ} \mathrm{C} .{ }^{1} \mathrm{H}$ NMR (300 MHz, $\left.\mathrm{CDCl}_{3}\right) \delta(\mathrm{ppm}): 8.58-8.39$ (m, 2H), $8.35(\mathrm{~s}, 1 \mathrm{H}), 8.19(\mathrm{~d}, J=8.4 \mathrm{~Hz}, 2 \mathrm{H}), 7.00(\mathrm{~d}, J=8.4 \mathrm{~Hz}, 4 \mathrm{H}), 6.14(\mathrm{~s}, 1 \mathrm{H}), 4.03(\mathrm{~s}, 2 \mathrm{H})$, $3.89(\mathrm{~d}, J=1.5 \mathrm{~Hz}, 6 \mathrm{H}), 3.19(\mathrm{~s}, 3 \mathrm{H}), 3.11-2.43(\mathrm{~m}, 5 \mathrm{H}), 2.10-0.70(\mathrm{~m}, 10 \mathrm{H}) . \mathrm{MS}(\mathrm{CI}) \mathrm{m} / z(\%)$ : $520.2\left([\mathrm{M}+\mathrm{H}]^{+}, 43\right)$.

$N_{1-(2-(b e n z y l a m i n o)-2-o x o e t h y l)-N_{4}}$ (2,6-bis(4-methoxyphenyl)pyrimidin-4-yl)- $N_{1}$-methylsuccinamide (7f). Yield: 31\%. Mp. $220-222{ }^{\circ} \mathrm{C} .{ }^{1} \mathrm{H}$ NMR (300 MHz, DMSO- $\left.d_{6}\right) \delta$ (ppm): 10.87 (s, 1H), $8.57(\mathrm{~s}, 1 \mathrm{H}), 8.42(\mathrm{~d}, J=8.3 \mathrm{~Hz}, 2 \mathrm{H}), 8.31(\mathrm{~s}, 1 \mathrm{H}), 8.12(\mathrm{~d}, J=8.3 \mathrm{~Hz}, 2 \mathrm{H}), 7.85-6.80(\mathrm{~m}, 9 \mathrm{H})$, $4.61-4.14(\mathrm{~m}, 2 \mathrm{H}), 4.11-3.89(\mathrm{~m}, 2 \mathrm{H}), 3.83(\mathrm{~s}, 6 \mathrm{H}), 3.05(\mathrm{~s}, 3 \mathrm{H}), 2.93-2.69(\mathrm{~m}, 4 \mathrm{H}) . \mathrm{MS}(\mathrm{CI})$ $m / z(\%): 560.1\left([\mathrm{M}+\mathrm{H}]^{+}, 82\right)$.

N-(2,6-bis(4-methoxyphenyl)pyrimidin-4-yl)-2-(2-((2-(tert-butylamino)-2-oxoethyl)amino)-2-oxoethoxy)acetamide (8a). Yield: 23\%. Oil. ${ }^{1} \mathrm{H}$ NMR (300 MHz, $\left.\mathrm{CDCl}_{3}\right) \delta(\mathrm{ppm}): 9.60(\mathrm{~s}, 1 \mathrm{H}), 8.50(\mathrm{~d}$, $J=8.3 \mathrm{~Hz}, 2 \mathrm{H}), 8.43(\mathrm{~s}, 1 \mathrm{H}), 8.30-8.15(\mathrm{~m}, 2 \mathrm{H}), 7.20-6.89(\mathrm{~m}, 4 \mathrm{H}), 6.00(\mathrm{~s}, 1 \mathrm{H}), 4.53(\mathrm{~s}, 2 \mathrm{H})$, $4.28(\mathrm{~s}, 2 \mathrm{H}), 3.89(\mathrm{~s}, 6 \mathrm{H}), 3.39(\mathrm{~s}, 2 \mathrm{H}), 1.34(\mathrm{~s}, 9 \mathrm{H}) .{ }^{13} \mathrm{C} \mathrm{NMR}\left(75 \mathrm{MHz}, \mathrm{CDCl}_{3}\right) \delta(\mathrm{ppm}): 170.3$, $168.8,167.3,165.3,163.7,161.9,161.8,157.6,130.4,129.9,129.9,129.0,114.1,113.7,101.8,71.6$, 69.5, 55.4, 52.1, 51.9, 28.7. MS (CI) $m / z(\%): 534.2\left([\mathrm{M}+\mathrm{H}]^{+}, 100\right)$.

N-(2,6-bis(4-methoxyphenyl)pyrimidin-4-yl)-2-(2-((2-(cyclohexylamino)-2-oxoethyl)amino)-2-oxoethoxy)acetamide (8b). Yield: 36\%. Mp. $113-115^{\circ} \mathrm{C} .{ }^{1} \mathrm{H} \mathrm{NMR}\left(300 \mathrm{MHz}, \mathrm{CDCl}_{3}\right) \delta(\mathrm{ppm}): 9.57$ (s, $1 \mathrm{H}), 8.50(\mathrm{~d}, J=8.3 \mathrm{~Hz}, 2 \mathrm{H}), 8.42(\mathrm{~s}, 1 \mathrm{H}), 8.22(\mathrm{~d}, J=8.7 \mathrm{~Hz}, 2 \mathrm{H}), 7.01(\mathrm{dd}, J=8.8,6.8 \mathrm{~Hz}, 4 \mathrm{H})$, 6.19 (brs, $J=8.0 \mathrm{~Hz}, 1 \mathrm{H}), 4.53(\mathrm{~s}, 2 \mathrm{H}), 4.27(\mathrm{~s}, 2 \mathrm{H}), 3.88(\mathrm{~s}, 6 \mathrm{H}), 3.81-3.55(\mathrm{~m}, 1 \mathrm{H}), 3.39(\mathrm{~s}, 2 \mathrm{H})$, $2.21-0.92(\mathrm{~m}, 10 \mathrm{H}) .{ }^{13} \mathrm{C} \mathrm{NMR}\left(75 \mathrm{MHz}, \mathrm{CDCl}_{3}\right) \delta(\mathrm{ppm}): 170.7,169.1,167.5,165.6,162.2,162.1$, $157.9,154.2,130.7,130.2,129.3,114.4,114.0,102.1,71.9,69.5,56.4,55.7,51.8,48.6,33.1,25.7$, 24.9. MS (CI) $m / z(\%): 561.5\left([\mathrm{M}+\mathrm{H}]^{+}, 100\right)$. 
N-benzyl-2-(2-(2-((2,6-bis(4-methoxyphenyl)pyrimidin-4-yl)amino)-2-oxoethoxy)acetamido) acetamide (8c). Yield: 25\%. Mp. $201-203{ }^{\circ} \mathrm{C} .{ }^{1} \mathrm{H}$ NMR (300 MHz, $\left.\mathrm{CDCl}_{3}\right) \delta(\mathrm{ppm}): 9.60$ (s, 1H), 8.49 $(\mathrm{d}, J=8.1 \mathrm{~Hz}, 2 \mathrm{H}), 8.39(\mathrm{~s}, 1 \mathrm{H}), 8.23(\mathrm{~d}, J=8.5 \mathrm{~Hz}, 2 \mathrm{H}), 7.50-7.11(\mathrm{~m}, 6 \mathrm{H}), 7.00(\mathrm{dd}, J=8.5,8.1$ $\mathrm{Hz}, 4 \mathrm{H}), 4.48$ (s, 2H), 4.40 (s, 2H), 4.24 (s, 2H), 4.07 (s, 2H), 3.88 (s, 6H). MS (CI) m/z (\%): 570.5 $\left([\mathrm{M}+\mathrm{H}]^{+}, 73\right)$.

2-(2-((2,6-bis(4-methoxyphenyl)pyrimidin-4-yl)amino)-2-oxoethoxy)-N-(2-(tert-butylamino)-2-oxoethyl)-N-methylacetamide (8d). Yield: 35\%. Mp. $115-117^{\circ} \mathrm{C} .{ }^{1} \mathrm{H}$ NMR (300 MHz, $\left.\mathrm{cdcl}_{3}\right) \delta(\mathrm{ppm})$ : $9.63(\mathrm{~s}, 1 \mathrm{H}), 8.49(\mathrm{~d}, J=8.9 \mathrm{~Hz}, 2 \mathrm{H}), 8.42(\mathrm{~s}, 1 \mathrm{H}), 8.22(\mathrm{~d}, J=8.8 \mathrm{~Hz}, 2 \mathrm{H}), 7.07-6.93(\mathrm{~m}, 4 \mathrm{H})$, $4.45(\mathrm{~s}, 2 \mathrm{H}), 4.28(\mathrm{~s}, 2 \mathrm{H}), 3.94(\mathrm{~d}, J=1.5 \mathrm{~Hz}, 2 \mathrm{H}), 3.88(\mathrm{~s}, 6 \mathrm{H}), 3.07(\mathrm{~s}, 3 \mathrm{H}), 1.35(\mathrm{~s}, 9 \mathrm{H}) .{ }^{13} \mathrm{C}$ NMR (75 MHz, DMSO- $\left.d_{6}\right) \delta(\mathrm{ppm}): 170.8,170.8,170.3,164.6,163.4,162.1,162.1,156.7,130.1,130.0$, $129.5,129.0,114.9,114.4,101.5,69.6,67.1,55.9,55.8,50.9,34.8,28.9$. MS (CI) $m / z(\%): 550.2$ $\left([\mathrm{M}+\mathrm{H}]^{+}, 100\right)$.

2-(2-((2,6-bis(4-methoxyphenyl)pyrimidin-4-yl)amino)-2-oxoethoxy)-N-(2-(cyclohexylamino)-2oxoethyl)-N-methylacetamide (8e). Yield: 37\%. Mp. $186-188{ }^{\circ} \mathrm{C} .{ }^{1} \mathrm{H} \mathrm{NMR}\left(300 \mathrm{MHz}, \mathrm{cdcl}_{3}\right) \delta$ (ppm): 9.69 (s, 1H), 8.50 (d, $J=8.5 \mathrm{~Hz}, 2 \mathrm{H}), 8.42(\mathrm{~s}, 1 \mathrm{H}), 8.22(\mathrm{~d}, J=8.6 \mathrm{~Hz}, 2 \mathrm{H}), 7.00(\mathrm{t}, J=8.1$ Hz, 4H), 4.44 (s, 2H), 4.26 (s, 2H), 3.99 (s, 2H), 3.88 (s, 6H), 3.76 (s, 1H), $3.14-3.01$ (m, 3H), 2.16 $-0.79(\mathrm{~m}, 10 \mathrm{H}) .{ }^{13} \mathrm{C} \mathrm{NMR}\left(75 \mathrm{MHz}, \mathrm{CDCl}_{3}\right) \delta(\mathrm{ppm}): 169.8,169.3,167.4,165.6,164.0,162.2$, $162.1,157.9,130.7,130.3,130.2,129.3,114.4,114.0,102.1,72.0,69.7,55.7,52.8,48.7,35.8,33.2$, 25.7, 25.0. MS (CI) $m / z(\%): 576.6\left([\mathrm{M}+\mathrm{H}]^{+}, 100\right)$.

N-(2-(benzylamino)-2-oxoethyl)-2-(2-((2,6-bis(4-methoxyphenyl)pyrimidin-4-yl)amino)-2-oxoethoxy)-N-methylacetamide (8f). Yield: 29\%. Mp. $166-168^{\circ} \mathrm{C} .{ }^{1} \mathrm{H}$ NMR $\left(300 \mathrm{MHz}, \mathrm{CDCl}_{3}\right) \delta(\mathrm{ppm})$ : $9.68(\mathrm{~s}, 1 \mathrm{H}), 8.62-8.43(\mathrm{~m}, 2 \mathrm{H}), 8.40(\mathrm{~s}, 1 \mathrm{H}), 8.31-8.13(\mathrm{~m}, 2 \mathrm{H}), 7.41-7.15(\mathrm{~m}, 5 \mathrm{H}), 7.10-6.89$ (m, 4H), $6.76-6.45(\mathrm{~m}, 1 \mathrm{H}), 4.55-4.27(\mathrm{~m}, 4 \mathrm{H}), 4.22(\mathrm{~d}, J=7.7 \mathrm{~Hz}, 2 \mathrm{H}), 4.08(\mathrm{~s}, 2 \mathrm{H}), 3.89(\mathrm{~s}$, 6H), $3.06(\mathrm{~s}, 3 \mathrm{H})$. MS (CI) $m / z(\%): 584.2\left([\mathrm{M}+\mathrm{H}]^{+}, 88\right)$. 
General procedure for the synthesis of N-(2,6-bis(4-methoxyphenyl)pyrimidin-4-yl)-4-(4-(2(alkylamino)-2-oxoethyl)piperazin-1-yl)-4-oxobutanamides $\quad(9 a-c) \quad$ and $\quad N$-(2,6-bis(4methoxyphenyl)pyrimidin-4-yl)-2-(2-(4-(2-(alkylamino)-2-oxoethyl)piperazin-1-yl)-2oxoethoxy)acetamides (10a-c)

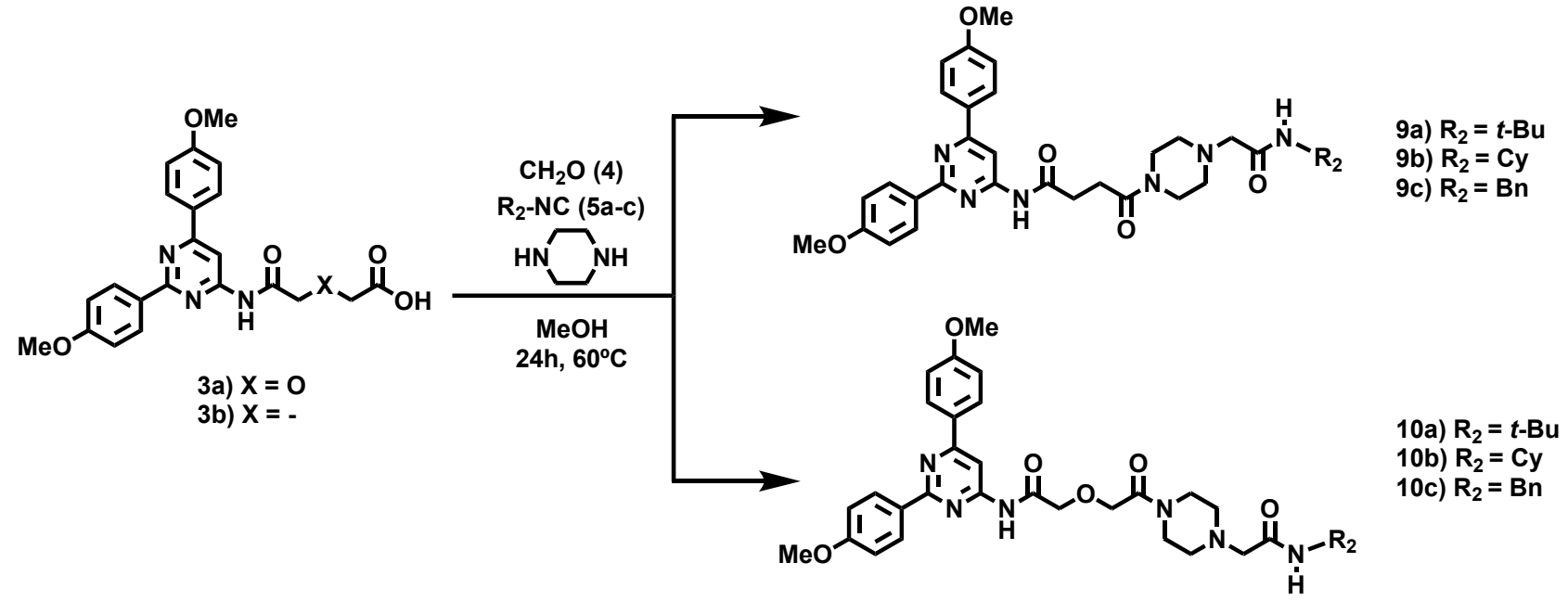

A mixture of the synthetised acid 3a-b (1 equiv.), formaldehyde 4 ( 1 equiv.), the isocyanide 5a-c (1 equiv.) and piperidine ( 1 equiv) in $\mathrm{MeOH}(3 \mathrm{~mL})$ was submitted to orbital stirring at $60^{\circ} \mathrm{C}$ for $24 \mathrm{~h}$. After completion of the reaction, $\mathrm{CH}_{2} \mathrm{Cl}_{2}(3 \mathrm{~mL})$ and PS- $p$ - $\mathrm{TsOH}$ (2 equiv.) were added. The reaction mixture was submitted to orbital stirring at room temperature until complete consumption of unreacted isocyanide (30-60 min). The polymeric reagent was filtered off and successively washed [2 times $(4 \mathrm{~mL})]$ with $\mathrm{MeOH}, \mathrm{AcOEt}$ and $\mathrm{CH}_{2} \mathrm{Cl}_{2}$. Evaporation of the solvents from the filtrate afforded a residue, treated with water and extracted with ethyl acetate [3 times $(15 \mathrm{~mL})]$. The organic phase was dried $\left(\mathrm{Na}_{2} \mathrm{SO}_{4}\right)$ and evaporated under reduced pressure to afford an oily residue that was purified by chromatographic methods on silica gel using hexane/AcOEt mixtures. 
N-(2,6-bis(4-methoxyphenyl)pyrimidin-4-yl)-4-(4-(2-(tert-butylamino)-2-oxoethyl)piperazin-1-yl)-4oxobutanamide (9a). Yield: 27\%. Mp. $203-205{ }^{\circ} \mathrm{C} .{ }^{1} \mathrm{H}$ NMR $\left(300 \mathrm{MHz}, \mathrm{CDCl}_{3}\right) \delta(\mathrm{ppm}): 8.92$ (s, $1 \mathrm{H}), 8.36(\mathrm{~s}, 1 \mathrm{H}), 8.31(\mathrm{~s}, 2 \mathrm{H}), 8.26-8.12(\mathrm{~m}, 2 \mathrm{H}), 7.08-6.94(\mathrm{~m}, 4 \mathrm{H}), 3.88(\mathrm{t}, J=1.9 \mathrm{~Hz}, 6 \mathrm{H})$, $3.68(\mathrm{t}, J=5.1 \mathrm{~Hz}, 2 \mathrm{H}), 3.53(\mathrm{t}, J=5.2 \mathrm{~Hz}, 2 \mathrm{H}), 3.03-2.70(\mathrm{~m}, 6 \mathrm{H}), 2.51(\mathrm{dt}, J=17.7,4.9 \mathrm{~Hz}, 4 \mathrm{H})$, $1.37(\mathrm{~d}, J=1.4 \mathrm{~Hz}, 9 \mathrm{H}) .{ }^{13} \mathrm{C} \mathrm{NMR}\left(75 \mathrm{MHz}, \mathrm{DMSO}-d_{6}\right) \delta(\mathrm{ppm}): 173.6,170.0,168.7,164.3,163.3$, $162.0,162.0,159.5,130.3,129.9,129.7,128.9,114.9,114.4,101.4,61.8,55.8,53.2,50.3,45.1,31.9$, 28.9, 27.7. HRMS (APCI) $m / z$ calcd for $\mathrm{C}_{32} \mathrm{H}_{41} \mathrm{~N}_{6} \mathrm{O}_{6}[\mathrm{M}+\mathrm{H}]^{+}:$589.3133; found: 589.3154 .

N-(2,6-bis(4-methoxyphenyl)pyrimidin-4-yl)-4-(4-(2-(cyclohexylamino)-2-oxoethyl)piperazin-1-yl)4-oxobutanamide (9b). Yield: 27\%. Oil. ${ }^{1} \mathrm{H}$ NMR (300 MHz, $\left.\mathrm{CDCl}_{3}\right) \delta(\mathrm{ppm}): 8.81$ (d, $J=2.3 \mathrm{~Hz}$, $1 \mathrm{H}), 8.50-8.39(\mathrm{~m}, 2 \mathrm{H}), 8.36(\mathrm{~d}, J=1.9 \mathrm{~Hz}, 1 \mathrm{H}), 8.25-8.13(\mathrm{~m}, 2 \mathrm{H}), 7.06-6.84(\mathrm{~m}, 4 \mathrm{H}), 3.87(\mathrm{~s}$, $6 \mathrm{H}), 3.85-3.74(\mathrm{~m}, 1 \mathrm{H}), 3.72-3.43(\mathrm{~m}, 4 \mathrm{H}), 3.01(\mathrm{~d}, J=2.1 \mathrm{~Hz}, 2 \mathrm{H}), 2.92-2.71(\mathrm{~m}, 2 \mathrm{H}), 2.71-$ $2.43(\mathrm{~m}, 6 \mathrm{H}), 1.89(\mathrm{~d}, J=12.2 \mathrm{~Hz}, 2 \mathrm{H}), 1.76-1.55(\mathrm{~m}, 4 \mathrm{H}), 1.39$ (q, $J=12.1 \mathrm{~Hz}, 2 \mathrm{H}), 1.30-1.07$ (m, 2H). MS (CI) $m / z(\%): 615.7\left([\mathrm{M}+\mathrm{H}]^{+}, 72\right)$.

4-(4-(2-(benzylamino)-2-oxoethyl)piperazin-1-yl)-N-(2,6-bis(4-methoxyphenyl)pyrimidin-4-yl)-4oxobutanamide (9c). Yield: 17\%. Mp. $194-195{ }^{\circ} \mathrm{C} .{ }^{1} \mathrm{H}$ NMR (300 MHz, $\left.\mathrm{CDCl}_{3}\right) \delta$ (ppm): 8.67 (s, $1 \mathrm{H}), 8.53-8.40(\mathrm{~m}, 2 \mathrm{H}), 8.37(\mathrm{~s}, 1 \mathrm{H}), 8.21(\mathrm{dd}, J=8.7,1.8 \mathrm{~Hz}, 2 \mathrm{H}), 7.52-7.13(\mathrm{~m}, 5 \mathrm{H}), 7.13-$ $6.84(\mathrm{~m}, 4 \mathrm{H}), 4.50(\mathrm{~d}, J=5.9 \mathrm{~Hz}, 2 \mathrm{H}), 3.90(\mathrm{~s}, 6 \mathrm{H}), 3.76-3.39(\mathrm{~m}, 4 \mathrm{H}), 3.25-3.10(\mathrm{~m}, 2 \mathrm{H}), 2.90-$ $2.69(\mathrm{~m}, 4 \mathrm{H}), 2.61(\mathrm{~s}, 4 \mathrm{H}) .{ }^{13} \mathrm{C} \mathrm{NMR}\left(75 \mathrm{MHz}, \mathrm{CDCl}_{3}\right) \delta(\mathrm{ppm}): 172.3,170.2,168.6,165.5,162.2$, $162.1,158.7,158.2,138.6,130.6,130.3,130.1,129.3,129.1,128.2,127.9,114.4,114.0,101.9,61.7$, 55.7, 53.6, 43.4, 42.0, 32.9, 28.5. HRMS (APCI) $m / z$ calcd for $\mathrm{C}_{35} \mathrm{H}_{39} \mathrm{~N}_{6} \mathrm{O}_{5}[\mathrm{M}+\mathrm{H}]^{+}$: 623.2976; found: 623.2985 . 
N-(2,6-bis(4-methoxyphenyl)pyrimidin-4-yl)-2-(2-(4-(2-(tert-butylamino)-2-oxoethyl)piperazin-1yl)-2-oxoethoxy)acetamide (10a). Yield: 28\%. Mp. $106-108{ }^{\circ} \mathrm{C} .{ }^{1} \mathrm{H}$ NMR $\left(300 \mathrm{MHz}, \mathrm{CDCl}_{3}\right) \delta$ (ppm): 99.64 (s, 1H), $8.54-8.42(\mathrm{~m}, 2 \mathrm{H}), 8.39(\mathrm{~s}, 1 \mathrm{H}), 8.27-8.14(\mathrm{~m}, 2 \mathrm{H}), 6.98$ (dd, $J=8.8,6.7$ $\mathrm{Hz}, 4 \mathrm{H}), 4.34(\mathrm{~s}, 2 \mathrm{H}), 4.24(\mathrm{~s}, 2 \mathrm{H}), 3.86(\mathrm{~s}, 6 \mathrm{H}), 3.68(\mathrm{q}, J=4.7 \mathrm{~Hz}, 2 \mathrm{H}), 3.38(\mathrm{t}, J=4.9 \mathrm{~Hz}, 2 \mathrm{H})$, $2.92(\mathrm{~s}, 2 \mathrm{H}), 2.52(\mathrm{t}, J=5.0 \mathrm{~Hz}, 4 \mathrm{H}), 1.38-1.30(\mathrm{~m}, 9 \mathrm{H})$. HRMS (APCI) $m / z$ calcd for $\mathrm{C}_{32} \mathrm{H}_{41} \mathrm{~N}_{6} \mathrm{O}_{6}$ $[\mathrm{M}+\mathrm{H}]^{+}:$605.3082; found: 604.3095 .

N-(2,6-bis(4-methoxyphenyl)pyrimidin-4-yl)-2-(2-(4-(2-(cyclohexylamino)-2-oxoethyl)piperazin-1yl)-2-oxoethoxy)acetamide (10b). Yield: 17\%. Oil. ${ }^{1} \mathrm{H}$ NMR (300 MHz, $\left.\mathrm{CDCl}_{3}\right) \delta(\mathrm{ppm}): 9.61$ (s, $1 \mathrm{H}), 8.48(\mathrm{~d}, J=8.6 \mathrm{~Hz}, 2 \mathrm{H}), 8.39(\mathrm{~s}, 1 \mathrm{H}), 8.21(\mathrm{~d}, J=8.5 \mathrm{~Hz}, 2 \mathrm{H}), 6.99(\mathrm{dd}, J=8.7,6.8 \mathrm{~Hz}, 4 \mathrm{H})$, $4.37(\mathrm{~s}, 2 \mathrm{H}), 4.19(\mathrm{~s}, 2 \mathrm{H}), 3.87(\mathrm{~s}, 6 \mathrm{H}), 3.74(\mathrm{~s}, 5 \mathrm{H}), 3.00(\mathrm{~d}, J=5.8 \mathrm{~Hz}, 2 \mathrm{H}), 2.67-2.37(\mathrm{~m}, 4 \mathrm{H})$, $2.26-0.20(\mathrm{~m}, 10 \mathrm{H}) .{ }^{13} \mathrm{C} \mathrm{NMR}\left(75 \mathrm{MHz}, \mathrm{CDCl}_{3}\right) \delta(\mathrm{ppm}): 168.8,168.0,166.9,165.3,163.7,161.9$, $161.8,157.6,130.4,129.9,129.0,114.1,113.7,101.8,71.6,69.6,61.5,55.4,55.4,53.2,47.5,44.4$, 41.9, 33.1, 33.0, 25.5, 24.7. HRMS (APCI) $m / z$ calcd for $\mathrm{C}_{34} \mathrm{H}_{43} \mathrm{~N}_{6} \mathrm{O}_{6}[\mathrm{M}+\mathrm{H}]^{+}:$631.3239; found: 631.3248 .

N-benzyl-2-(4-(2-(2-((2,6-bis(4-methoxyphenyl)pyrimidin-4-yl)amino)-2-oxoethoxy)acetyl)piperazin -1-yl)acetamide (10c). Yield: 28\%. Oil. ${ }^{1} \mathrm{H}$ NMR (300 MHz, $\left.\mathrm{CDCl}_{3}\right) \delta(\mathrm{ppm}): 9.58(\mathrm{~s}, 1 \mathrm{H}), 8.48(\mathrm{~d}$, $J=8.7 \mathrm{~Hz}, 2 \mathrm{H}), 8.40(\mathrm{~s}, 1 \mathrm{H}), 8.22(\mathrm{~d}, J=8.9 \mathrm{~Hz}, 2 \mathrm{H}), 7.48-7.12(\mathrm{~m}, 5 \mathrm{H}), 7.12-6.85(\mathrm{~m}, 4 \mathrm{H}), 4.48$ $(\mathrm{s}, 2 \mathrm{H}), 4.36(\mathrm{~s}, 2 \mathrm{H}), 4.25(\mathrm{~s}, 2 \mathrm{H}), 3.88(\mathrm{~s}, 6 \mathrm{H}), 3.72-3.54(\mathrm{~m}, 2 \mathrm{H}), 3.43-3.29(\mathrm{~m}, 2 \mathrm{H}), 3.08(\mathrm{~d}, J$ $=19.1 \mathrm{~Hz}, 2 \mathrm{H}), 2.69-2.42(\mathrm{~m}, 4 \mathrm{H}) .{ }^{13} \mathrm{C} \mathrm{NMR}\left(75 \mathrm{MHz}, \mathrm{CDCl}_{3}\right) \delta(\mathrm{ppm}): 169.2,168.8,166.9,165.3$, 163.7, 161.9, 161.8, 157.6, 138.2, 130.4, 129.9, 129.0, 128.8, 128.7, 127.6, 127.6, 127.5, 114.1, 113.7, 101.8, 71.6, 69.6, 61.5, 55.4, 55.4, 53.3, 53.1, 44.4, 43.1. MS (CI) $m / z(\%): 639.7\left([\mathrm{M}+\mathrm{H}]^{+}, 100\right)$. 


\section{Pharmacology. Binding assays}

The adenosinergic profile (affinity and selectivity) of the 18 novel pyrimidine derivatives (7-10) was evaluated in vitro (four human $\mathrm{AR}$ subtypes) using radioligand binding assays. Human adenosine receptors were expressed in transfected $\mathrm{CHO}\left(\mathrm{A}_{1} \mathrm{AR}\right), \mathrm{HeLa}\left(\mathrm{A}_{2 \mathrm{~A}} \mathrm{AR}\right.$ and $\left.\mathrm{A}_{3} \mathrm{AR}\right)$ and $\mathrm{HEK}-293\left(\mathrm{~A}_{2 \mathrm{~B}} \mathrm{AR}\right)$ cells. $\left({ }^{3} \mathrm{H}\right)-1,3-D i p r o p y l-8$-cyclopentylxanthine $\left(\left[{ }^{3} \mathrm{H}\right] \mathrm{DPCPX}\right)$ for $\mathrm{A}_{1} \mathrm{AR}$ and $\left[{ }^{3} \mathrm{H}\right] \mathrm{NECA}$ for $\mathrm{A}_{3} \mathrm{AR}$ were employed as radioligands in the assays. The biological data are expressed as $\mathrm{K}_{i}(\mathrm{nM}, \mathrm{n}=3)$ or as percentage inhibition of specific binding at $1 \mu \mathrm{M}(\mathrm{n}=2$, average $)$ for those compounds that did not fully displace specific radioligand binding. $\mathrm{K}_{i}$ values were obtained by fitting the data with nonlinear regression using Prism 2.1 software (GraphPad, San Diego, CA).

Furthermore, for $\mathrm{A}_{3} \mathrm{AR}$, fluorescence polarization binding experiments were carried out in a 96-well

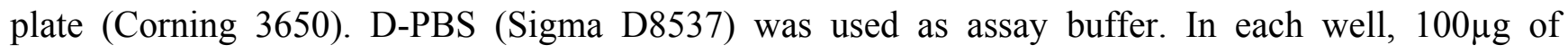
membranes from Hela-A3 cell line previously prepared in our laboratory (Lot: A006/ 17-01-2020, protein concentration $=3408 \mu \mathrm{g} / \mathrm{ml}$ ) were preincubated for 10 minutes at $\mathrm{RT}$ along with compounds used as standard and non-specific binding determined in presence of R-PIA 10 $\mu \mathrm{M}$ (Sigma S739). $75 \mathrm{nM}$ of the fluorescent ligand (CELT-228) was added, and the reaction mixture $(\mathrm{Vt}=200 \mu \mathrm{l})$ was incubated for 30 minutes at RT. The polarized fluorescence signal was read in an Infinite M1000 pro multiplate reader (Tecan 30063849). For validation of fluorescence polarization binding assay MRS-1220 $\left(h \mathrm{~A}_{3} \mathrm{AR} \mathrm{K}_{i}\right.$ described: $1.7 \mathrm{nM}$ ) was used as a control, obtaining a $h \mathrm{~A}_{3} \mathrm{AR}_{i}$ of $1.4 \mathrm{nM}$. The graphic below shows a representation of the concentration-response curves of the MRS-1220.

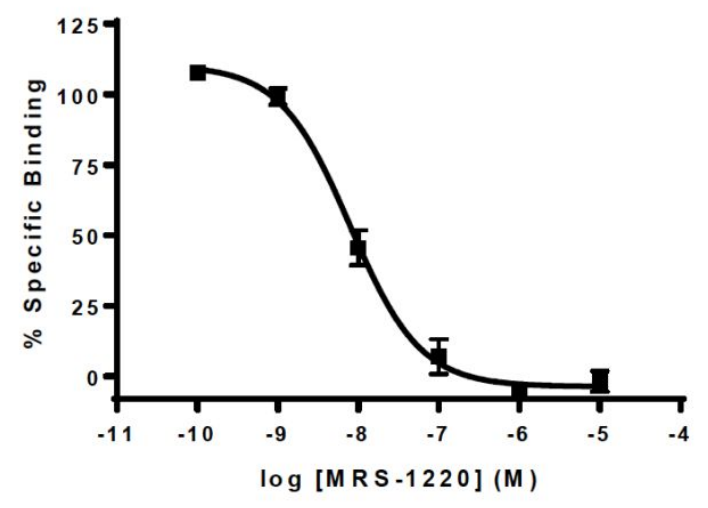




\section{HPLC traces of best compounds}

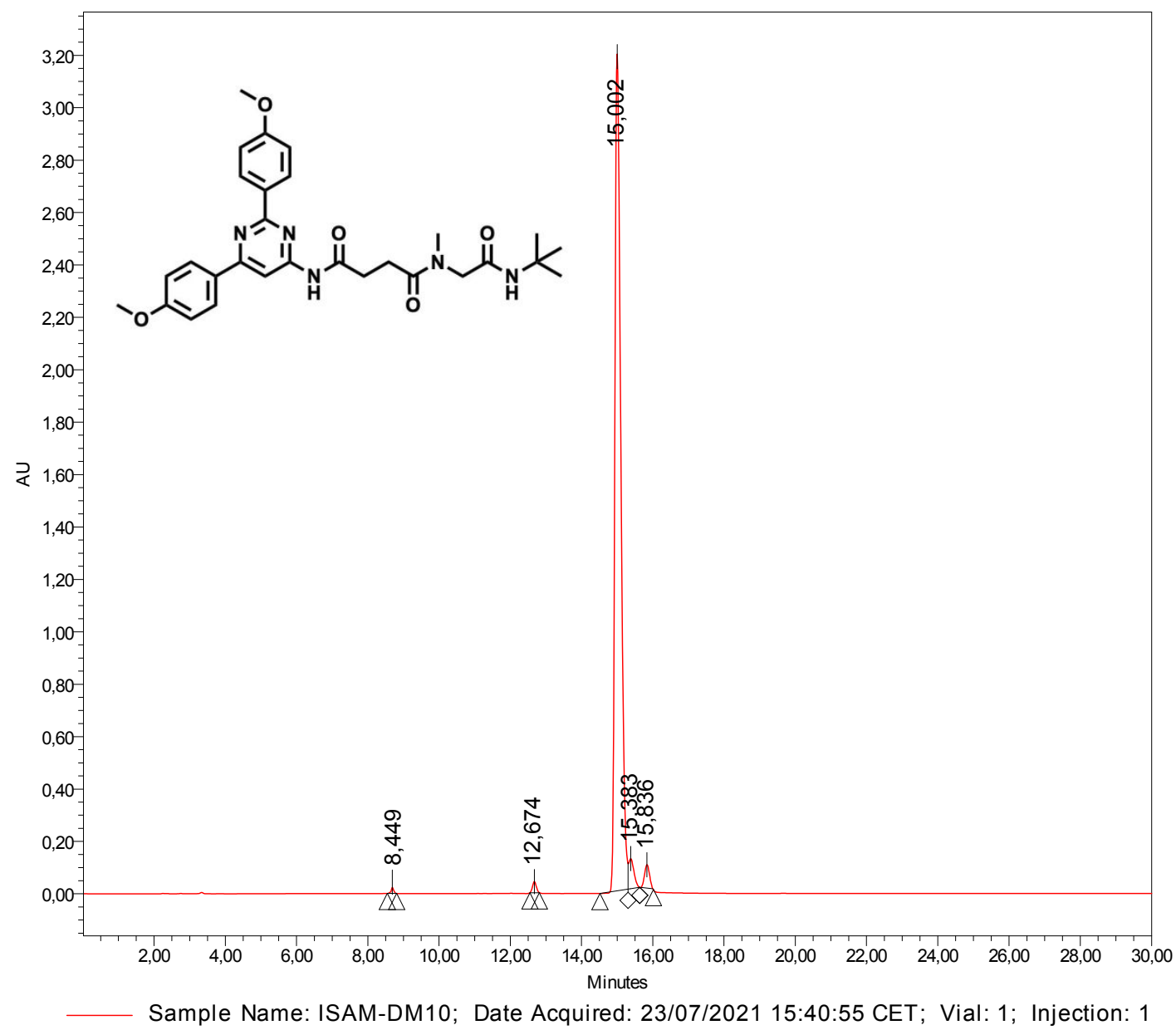

Peak Summary with Statistics

\begin{tabular}{|c|c|c|c|c|c|}
\hline \multicolumn{1}{|c|}{$\begin{array}{c}\text { Sample } \\
\text { Name }\end{array}$} & Vial & Inj. & $\begin{array}{c}\text { RT } \\
\text { (min) }\end{array}$ & $\%$ Area \\
\hline 1 & syldm-10 & 1 & 1 & 8,449 & 0,01 \\
\hline 2 & sy1dm-10 & 1 & 1 & 12,674 & 0,80 \\
\hline 3 & sy1dm-10 & 1 & 1 & 15,836 & 1,89 \\
\hline 4 & sy1dm-10 & 1 & 1 & 15,383 & 2,09 \\
\hline 5 & syldm-10 & 1 & 1 & 15,002 & 95,21 \\
\hline
\end{tabular}




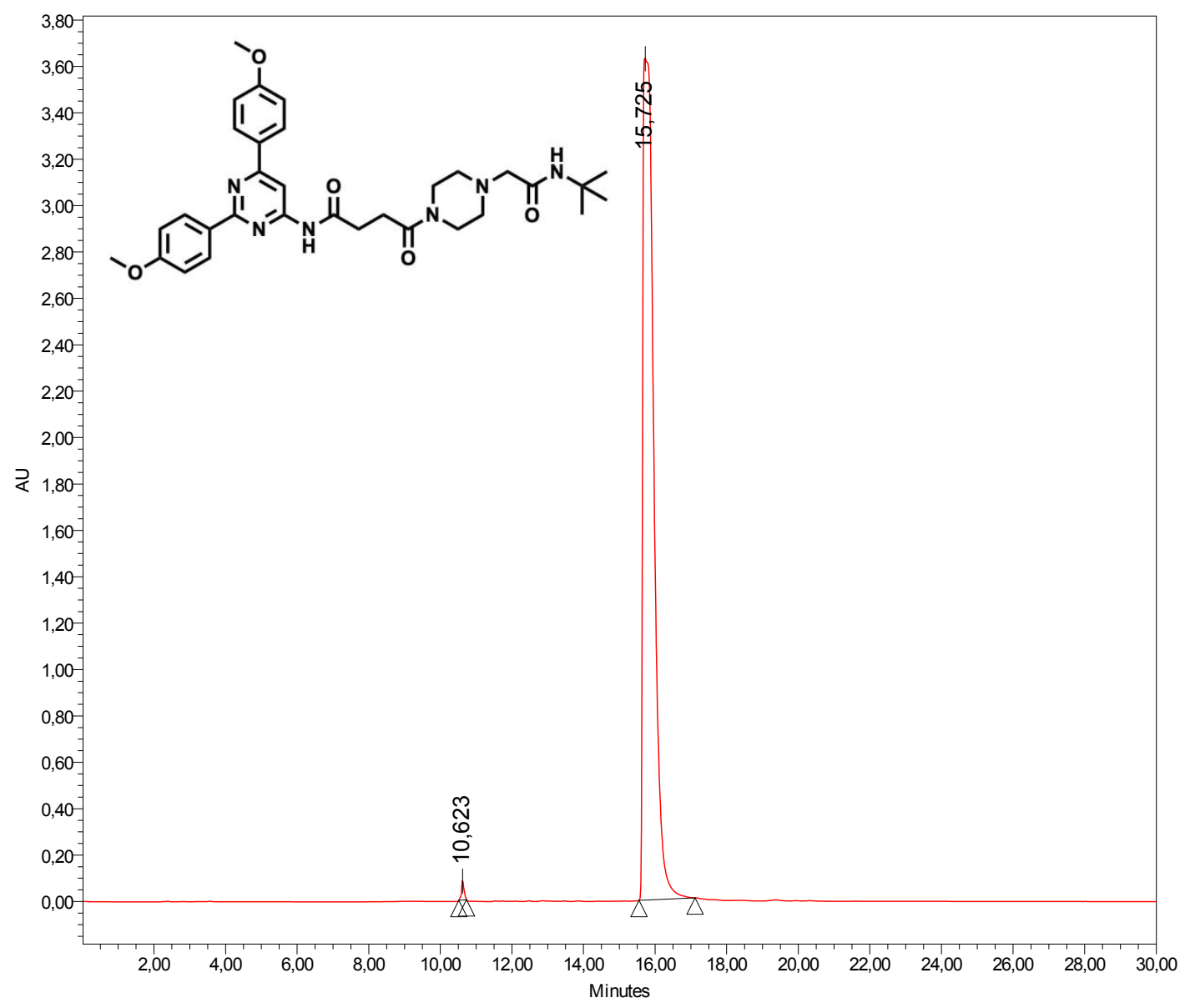

Sample Name: ISAM-DM13; Date Acquired: 23/07/2021 15:06:40 CET; Vial: 1; Injection: 1

Peak Summary with Statistics

Peak Name:

\begin{tabular}{|c|c|l|r|r|r|r|r|}
\hline & $\begin{array}{c}\text { Sample } \\
\text { Name }\end{array}$ & Vial & Inj. & $\begin{array}{c}\mathrm{RT} \\
(\mathrm{min})\end{array}$ & $\begin{array}{c}\text { Area } \\
\left(\mu \mathrm{V}^{*} \mathrm{sec}\right)\end{array}$ & $\%$ Area & $\begin{array}{c}\text { Height } \\
(\mu \mathrm{V})\end{array}$ \\
\hline 1 & sy1dm-13 & 1 & 1 & 15,725 & 77348717 & 99,46 & 3634316 \\
\hline 2 & sy1dm-13 & 1 & 1 & 10,623 & 419588 & 0,54 & 82024 \\
\hline
\end{tabular}




\section{Instrument Method: 10\%DCMiPAGradF1}

Stored: 23/07/2021 11:26:08 CET

\section{Method Information}

Method Modified User Breeze

Method Version

Method Edit User

\begin{tabular}{|c|c|c|c|c|c|c|}
\hline \multicolumn{7}{|c|}{ PCM15xx Instrument Setup } \\
\hline \multicolumn{3}{|l|}{ Type } & \multicolumn{3}{|c|}{ PCM/15xx } & $\mathrm{Hig}$ \\
\hline \multicolumn{3}{|c|}{ Instrument Status } & \multicolumn{3}{|l|}{ On } & Lov \\
\hline \multicolumn{3}{|c|}{ Pump Mode } & \multicolumn{3}{|c|}{ Gradient } & ot \\
\hline \multicolumn{3}{|c|}{ Flow A } & \multicolumn{3}{|c|}{1,00} & $s e$ \\
\hline \multicolumn{3}{|c|}{ Flow B } & \multicolumn{3}{|c|}{0,00} & ol \\
\hline \multicolumn{3}{|c|}{ Flow C } & \multicolumn{3}{|c|}{0,00} & Sol \\
\hline \multicolumn{7}{|c|}{ PCM/15xx Gradient Table } \\
\hline & Time & Flow & $\% A$ & $\% \mathrm{~B}$ & $\% \mathrm{C}$ & Curve \\
\hline 1 & & 1,00 & 100,0 & 0,0 & 0,0 & \\
\hline 2 & 30,00 & 1,00 & 90,0 & 10,0 & 0,0 & 6 \\
\hline
\end{tabular}

\begin{tabular}{ll}
\multicolumn{1}{c}{ W2487 Instrument Setup } \\
Type & W2487 \\
Instrument Status & On \\
Dual Wavelength & True \\
Pulse Period Seconds & 1,0 \\
Pulse Repeat Period Seconds & 1,0
\end{tabular}

W2487 Channel Information

Channel Name 2487Channel 1

Description $254 \mathrm{~nm}$

Use Channels On

Wavelength 254

Output Mode Absorbance A (Ch1)

Data Mode Absorbance A (Ch1)

Sampling Rate 1

Filter Type Hamming

Aufs $\quad 2,0000$

Time Constant $\quad 1,0$

AU Offset $\quad 0,000$

W2487 Channel Information

Channel Name 2487Channel 2

Description $280 \mathrm{~nm}$

Use Channels On

\begin{tabular}{l|l}
4000,0 & Solvent C \\
0,0 & \\
1,00 & \\
Off & \\
DCM & \\
iPA &
\end{tabular}

Voltage Offset $\quad 0$

Polarity +

AutoZero Wavelength True

AutoZero Keypad True

AutoZeroEvent Input True

AutoZero Inject True

Chart Mark Enable True

Ratio AuMinimum $\quad 0,1000$

Minimum Ratio $\quad 0,00$

Maximum Ratio $\quad 2,00$

Wavelength 280

Output Mode Absorbance B (Ch2)

Data Mode Absorbance B (Ch2) 


\section{References}

(1) Yaziji, V.; Rodríguez, D.; Gutiérrez-De-Terán, H.; Coelho, A.; Caamaño, O.; García-Mera, X.; Brea, J.; Loza, M. I.; Cadavid, M. I.; Sotelo, E. Pyrimidine derivatives as potent and selective $\mathrm{A}_{3}$ adenosine receptor antagonists. $J$. Med. Chem. 2011, 54 (2), 457-471. 